\title{
Effect of vitamin D level and polypharmacy on the risk of falls in the elderly
}

\author{
Gamze Dilek $^{1}$, Yalkin Calik $^{1}$, Kagan Ozkuk $^{2}$ \\ ${ }^{I}$ Department of Physical Medicine and Rehabilitation, BAIB Physical Medicine and Rehabilitation Hospital, Bolu, Turkey \\ ${ }^{2}$ Department of Medical Ecology and Hydroclimatology, Uşak University, Faculty of Medicine, Uşak, Turkey
}

\begin{abstract}
Aim: To investigate the effects of polypharmacy and vitamin D levels on the risk of falls in the elderly.

Methods: The prospective study included 201 patients (F/M: 155/46) aged 65 years and older who presented with nonspecific musculoskeletal pain. The demographic and laboratory data of the patients, as well as the results of a single leg stance test (SLST), a timed up and go (TUG) test and levels of vitamin D were recorded. Results: The percentage of patients with polypharmacy is 15.9 percent and 29.4 percent used no medications. The SLST score was the lowest and the TUG test score was significantly higher in the polypharmacy group $(p<0.05)$. Vitamin D levels were significantly higher in patients with normal SLST times than those with abnormal SLST times $(p<0.05)$. The risk of falls was significantly higher among patients with a previous history of a fall $(p<0.05)$. Polypharmacy and the female gender appeared as the most significant factors affecting the risk of falls $(p<0.05)$, while vitamin D level was found to have no effect $(p>0.05)$.

Conclusion: Medical therapies for the treatment of diseases in the elderly should have a rational basis, as this may reduce falls, particularly in the elderly population, that can have serious consequences, and may even lead to death.
\end{abstract}

Keywords: Accidental falls, elderly, risk factors, polypharmacy, vitamin D deficiency.

$\triangle$ Dr. Gamze Dilek

Department of Physical Medicine and Rehabilitation, BAIB Physical Medicine and Rehabilitation Hospital, Bolu, Turkey

E-mail: gamzedilekk@gmail.com

Received: 2020-10-03 Revised: 2020-11-09

Accepted: 2020-12-07 / Published online: 2021-04-01

\section{Introduction}

The aging global population has brought problems related to health services, the economy and the environment. Falls among the elderly may result from impaired vision, vestibular and proprioceptive sensation, and muscle strength [1]. Falls can occur as a result of distruption to postural balance, which ensures that the center of gravity remains in contact with the support surface, and can cause serious mortality and morbidity [2]. Among elderly people aged 65 years and older, approximately 13 percent have a balance disorder and one-third experience at least one fall per year [3]. In case of fall, 20 percent of people are admitted to hospital and 10 percent may have soft tissue or joint damage, or even fractures [4]. Studies have shown that there are many factors other than age that increase the risk of falls, these include multiple chronic disorders (musculoskeletal disorders, neurologic and psychotic disorders, diabetes mellitus, etc.), polypharmacy and vitamin D deficiencies [5,6].

The most common definition of polypharmacy in studies refers to the use of four or more medications in one day [7]. Medications can 
have side effects related to the metabolic state already affected by aging, and these side effects may increase the risk of falls. Researchers can be divided into two different groups, based on the results of a meta-analysis on this subject. While some emphasize the importance of a large number of medications, others place more emphasis on the type of medications used (antiaritmic, antihypertensive and antiepileptic drugs, etc.) [8]. There are also studies indicating that polypharmacy contributes two times more to falls than any other factor [9], while other studies have suggested that the use of at least one drug that contributes to falls has a more significant impact [10].

Epidemiological studies have identified a possible association between vitamin D level and risk of falling [11], although there is controversy between such studies. Some more recent studies suggest that vitamin $\mathrm{D}$ regulates muscle development, its deficiency may cause muscle weakness and may be associated with an increased risk of falls in the elderly [12]. Other studies, in contrast, found no association between falls and vitamin D level [13].

Studies on polypharmacy and vitamin D levels have given conflicting results in terms of their effects on falls. The aim of this study is to investigate whether polypharmacy and vitamin D levels affect the risk of falls in any way.

\section{Materials and Methods}

Patients aged 65 years and older who were hospitalized for nonspecific muscle pain within the previous year were prospectively studied. Ethical approval was obtained from Clinical Trials Ethical Board at Bolu Abant Baysal University (Date and decision number: 2015/64). Demographic data of the patients, laboratory and X-ray results, history of falls in the last year, comorbid diseases and the number of medications being used were recorded.
Patients with total blood count, erythrocyte sedimentation rate and $\mathrm{C}$-reactive protein levels within normal ranges are included, whereas those with scoliosis, degenerative and inflammatory joint disease in the lower extremity, total hip and/or knee prosthesis, neuromuscular disease, dementia, mental retardation, traumatic brain damage, cerebrovascular disease, spinal cord injury, severe cardiovascular disease, visual impairment, and patients with alcohol and drug addictions were excluded from the study. Polypharmacy was defined as the use of four or more medications, and vitamin D deficiency was defined as $25(\mathrm{OH}) \mathrm{D} 3$ levels below 30 $\mathrm{ng} / \mathrm{ml}$, while values of $30 \mathrm{ng} / \mathrm{ml}$ and higher were defined as normal [14]. Single-leg stance test (SLST) and the timed up and go (TUG) test results were evaluated to determine the risk of falling. In the SLST, patients were instructed to stand on one leg hands facing down by their sides. The test was terminated when the patient's foot touched the ground, changed position or received support from the ground. The test lasts a maximum of 30 seconds and is performed three times, with the best performance recorded for analysis. A test result of 5 seconds or less indicates a balance disorder. In the TUG test, patients are instructed to get up from a chair without support, walk three meters and turn back, return to the chair, and then sit down. A test result of 14 seconds or higher indicates a balance disorder.

\section{Statistical analysis}

The statistical analysis was performed using the SPSS 22.0 statistical software (IBM Corp., Armonk, NY, US). A Shapiro-Wilk Test was used to determine whether the data was normally distributed. Data with a normal distribution was expressed as mean \pm standard deviation, while data without normal distribution was expressed as a median (min, 
max). Paired correlations were evaluated from a Spearman's rank correlation coefficient, and a Mann-Whitney U test was used to evaluate the relationship between two independent groups, A Kruskal-Wallis test was used to evaluate the relationship between more than two independent groups, a Chi-Square test to evaluate the relationship between categorical variables, and the proportion of the categorical groups was expressed as a percentage (\%). The effects of the quantitative and categorical data on the other groups were evaluated by logistic regression analysis. A $\mathrm{p}$ value of $<0.05$ was considered statistically significant.

\section{Results}

The study included 201 patients (F/M: 155/46) with a mean age of $68.6(65-75)$ years (Table 1). It was found that 15.9 percent of the study patients used polypharmacy and 29.4 percent were not on any medications. 51.7 percent of the patients using medication used antihypertensive drugs, 6.0 percent used antidiabetic and 2.5 percent used antipsychotic medications. The SLST duration was normal in 49.3 percent and the TUG test was normal in 60.7 percent of the participants. There was a significant negative correlation between the SLST and TUG test results $(r:-256, p<0.05)$, and also a significant correlation between the number of drugs and SLST duration $(p<0.05)$.

Table 1. Demographic values.

\begin{tabular}{|l|c|l|c|}
\hline Variables & Median & Min. & Max. \\
\hline Age (year) & 68,6 & 65 & 75 \\
\hline $\begin{array}{l}\text { Body mass index } \\
\left(\mathrm{kg} / \mathrm{m}^{2}\right)\end{array}$ & 25,9 & 22,1 & 27,5 \\
\hline $\begin{array}{l}\text { Vitamin D } \\
\text { (IU/ml) }\end{array}$ & 18,3 & 3 & 97,3 \\
\hline $\begin{array}{l}\text { Single-leg } \\
\text { distance test } \\
\text { (SLDT) (sn) }\end{array}$ & 12,6 & 4 & 21 \\
\hline $\begin{array}{l}\text { Time up \& go test } \\
\text { (TGT) (sn) }\end{array}$ & 5,6 & 0 & 15 \\
\hline
\end{tabular}

SLST duration was observed to decrease with the increasing number of medications, and SLST durations were found significantly shorter in the polypharmacy group compared to patients who received less than four medications $(p<0.05)$, (Figure 1).

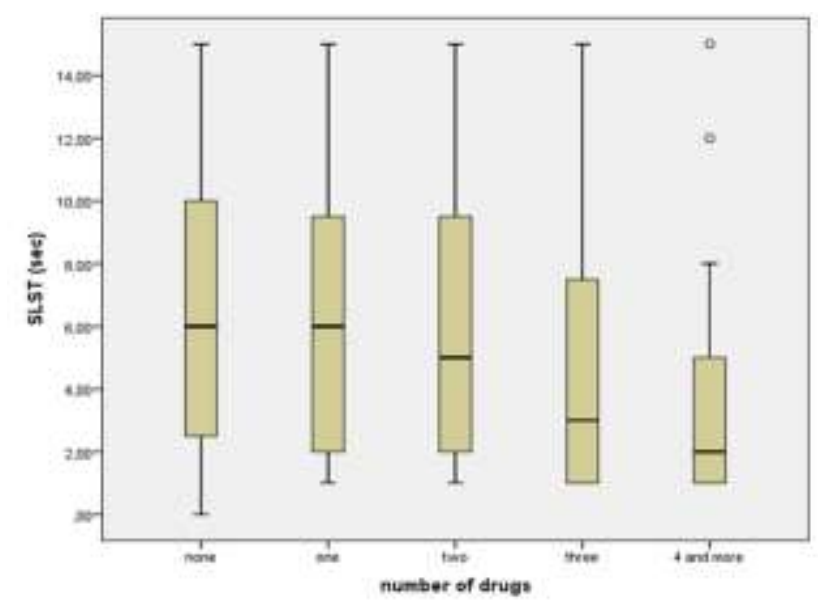

Figure 1. Relationship between SLST duration and number of drugs.

Concomitant use of more than one drug was more common in females then males. Of the study group, 29.8 percent had a remarkable history of falls, and the falling rate was significantly higher in females $(88.3 \%)$ than in males $(72.3 \%)(p<0.05)$. SLST times were significantly lower and TUG times were higher in patients with a history of falling compared to those without a history of falling $(p<0.05)$, (Figure 2 and 3 ).

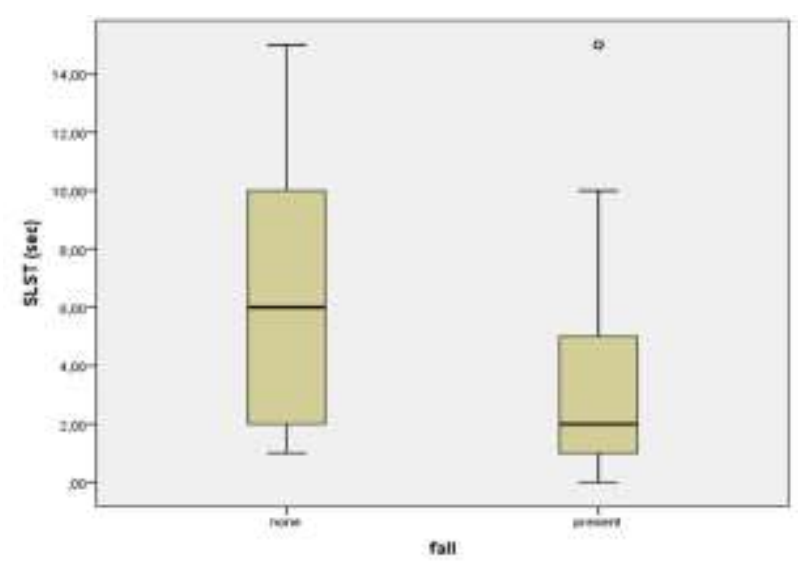

Figure 2. Relationship between falls and SLST duration. 


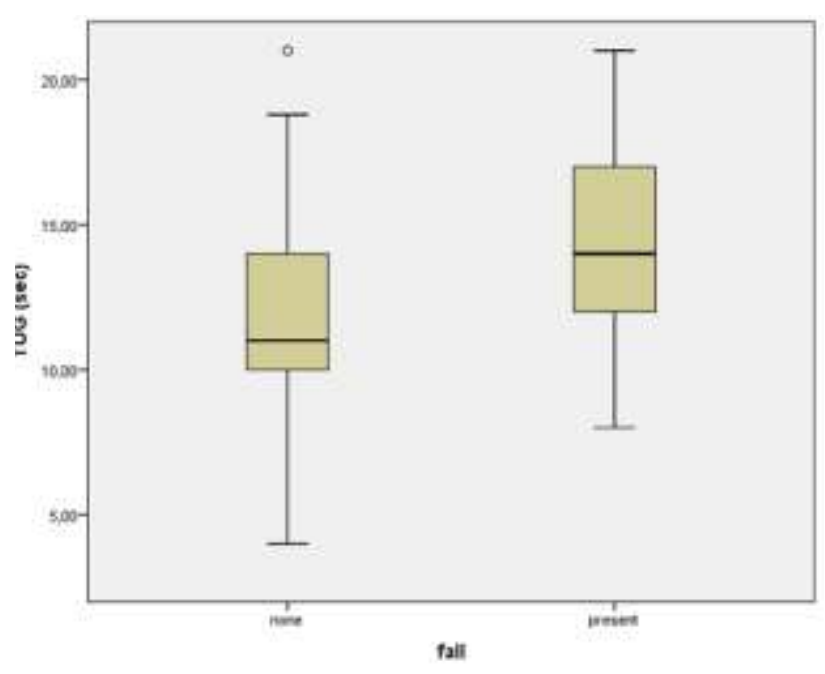

Figure 3. Relationship between falls and TUG duration.

SLST durations were significantly shorter and TUG durations were longer in patients using antipsychotic medications when compared to those who did not use antipsychotic medications $(p<0.05)$. Only the SLST time was significantly shorter in patients on antidiabetics when compared to patients who did not take antidiabetic medication ( $p<0.05)$, (Figure 4$)$.

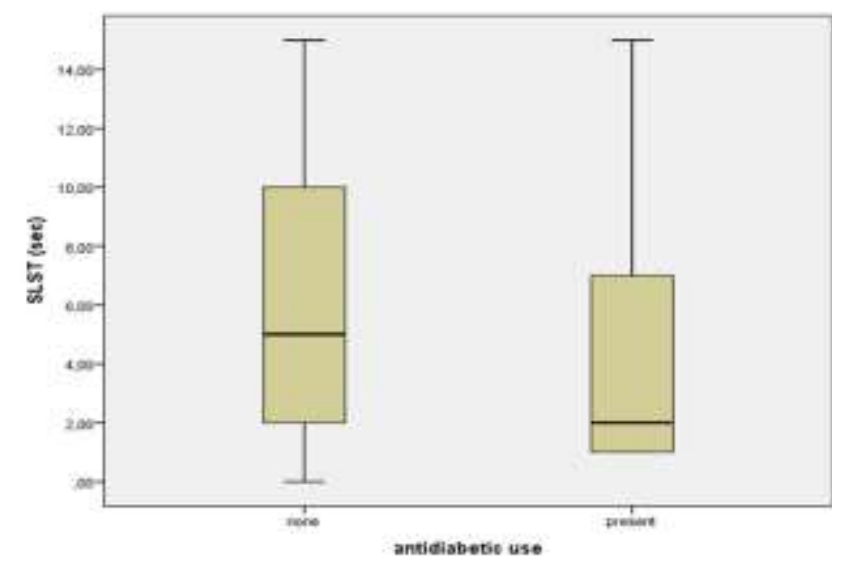

Figure 4. Relationship between antidiabetic use and SLST duration.

SLST durations were significantly lower and TUG durations were higher in female patients compared to male patients $(p<0.05)$. There was a significant positive correlation between vitamin D levels and SLST duration $(r: 0.212$ $p<0.05)$, and vitamin D levels were significantly higher in patients with normal SLST times than those with abnormal SLST times $(p<0.05)$. In the evaluation of the factors affecting the risk of falls, polypharmacy followed by TUG time and female gender had the most significant positive effects (Table 2) $(p<0.05)$.

Table 2. The factors effecting falls.

\begin{tabular}{|c|c|c|c|c|}
\hline \multirow[t]{2}{*}{ Variables } & \multirow[t]{2}{*}{$\boldsymbol{\beta}$} & \multicolumn{2}{|c|}{$\begin{array}{c}95 \% \\
\text { Confidence } \\
\text { Interval }\end{array}$} & $p$ \\
\hline & & Min. & Max. & \multirow{4}{*}{$<0,05$} \\
\hline $\begin{array}{l}\text { Number of } \\
\text { drugs }\end{array}$ & 3,66 & 1,08 & 12,42 & \\
\hline $\begin{array}{l}\text { Time up \& } \\
\text { go test (sn) }\end{array}$ & 1,24 & 1,10 & 1,39 & \\
\hline $\begin{array}{l}\text { Women } \\
\text { gender }\end{array}$ & 0,35 & 0,13 & 0,96 & \\
\hline
\end{tabular}

\section{Discussion}

Showing an increasing prevalence with increased life expectancy, approximately 30-40 percent of elderly people aged 65 years and older experience falls, and 50 percent of these patients have a history of recurrent falls. Preventive measures must be taken due to the increased morbidity and mortality risk in the elderly after falling [15]. In population-based studies, researchers have reported rates of 16.5 percent, 21.8 percent and 45.4 percent for recurrent falls $[4,5,9]$ compared to the recurrent falling rate of 29.9 percent in the this study. Studies have also found that females are three times more likely to experience falls than men, which has been attributed to the difference in muscle mass due to gender, the use of high heels in women and more common household accidents among women. In the present study, recurrent falls were twice as common in women as in men [7].

Due to the potentially serious consequences of falls, studies have focused on determining risk factors and in developing strategies to prevent 
falls. Simply, falls occur when an individual loses his/her balance, and therefore balance assessments are the most important and relevant in the prevention of falls. Researchers have developed several tests for balance assessment, including the SLST and TUG tests which observe static and dynamic balance [16]. A three-year study involving 316 volunteers established SLST time as a risk factor for falls, and reported an association between recurrent falls, whereas another study involving patients with a mean age of 73.6 years reported no such relationship [17]. In the present study, the mean age of the study population was 68.6 years and SLST times were significantly lower in patients with a history of recurrent falls. Studies involving patients aged 65 years and above reported a relationship between TUG time and age, and suggested that a high TUG time is associated with recurrent falls [10]. The present study found no significant relationship between TUG time and age, whereas a significant relationship was noted between TUG time and repeated falls.

Hypertension, diabetes, and neurological and psychological disorders are the leading comorbid conditions that increase in parallel with aging. In recent years, there has been a linear increase in the use of antihypertensive, antidiabetic and antipsychotic drugs among the increasing elderly population [10]. Kojima et al. [9] evaluated the two-year fall rate in patients older than 65 years taking medication for chronic diseases, and they found that the concurrent use of multiple medications increased the prevalence of falls. In a longitudinal study, Dhalwani et al. [18] reported a rate of falls of 18 percent in subjects who were on four or more medications, and 50 percent in subjects on 10 of more medications. There are many studies reporting a significant negative correlation between the number of medications and SLST time, whereas other studies have found that certain types of medication (antihypertensive, antidiabetic, antipsychotic) are more likely to increase falls [8]. These studies define such medications as drugs that increase the risk of falls. The rate of polypharmacy was found to be as high as 79.9 percent in a study involving 293 elderly hospice patients, and polypharmacy was found to increase the rate of fall-related injuries 4.5 times [4], although another study reported that polypharmacy increases the rate of injury 1.5 times [3]. In the present study, the risk of falls increased 3.6 times. Studies have also shown that the use of medications that increase the risk of falls (antihypertensive, antidiabetic, antipsychotic) bring about a further increase in risk when combined with polypharmacy. In a prospective study evaluating patients aged 55 years and above who were admitted to hospital, Ziere et al. [7] reported an increased risk of falls among patients who used four or more medications, and stated that the use of even a single medication increased the rate of falls in the presence of a drug that increased the risk of falls. Laflamme et al. [19] retrospectively evaluated patients aged 65 years and above who presented with fall-related injuries, and found that the risk of falls increased in the presence of polypharmacy, although an increased risk of falls was identified in the presence of medication use that increased the risk of falls regardless of the number of medications. RA balance study involving patients with hypertension found that the use of antihypertensive medications had a negative effect on balance [20], while the present study, in contrast, found no such effect of antihypertensive medication use on the risk of falls, the rate of which was 73 percent in the polypharmacy group. This is attributed to the widespread use of antihypertensive therapies, 
while similar studies have found that patients on antidiabetic and antipsychotic medication are more likely to experience falls. Similarly, the present study found a significantly higher rate of antidiabetic and antipsychotic medication use in the polypharmacy group, indicating its negative effect on balance. Taking drug use into consideration rationally would prevent possible drug side effects and would reduce their negative effects on the risk of falls. The musculoskeletal system is another body system that affects balance. Maintaining sufficient level of minerals and vitamins in the body has been found to be associated with overall health status and balance mechanisms, and vitamin $\mathrm{D}$ is one of the more important vitamins in this regard [13]. Vitamin D acts as a precursor hormone analogue, and plays an important role in several important mechanisms, including calcium imbalance [13]. Studies have found impaired balance and muscle development in patients with vitamin D deficiency, indicating that vitamin D levels have a positive effect on physical performance in individuals aged 65 years and above [6]. It has been reported that elderly people with normal vitamin $\mathrm{D}$ levels perform better than people with vitamin $\mathrm{D}$ deficiency, with vitamin D levels below $24 \mathrm{ng} / \mathrm{ml}$ in particular have dramatic negative effects on balance, leading to recommendations for careful attention to vitamin D levels [21]. SLST times were higher in individuals aged 60 years and older with normal vitamin D levels than patients with lower levels [13], and the present study found significantly lower TUG durations in patients with vitamin $\mathrm{D}$ deficiency, although no effect of vitamin D was observed on falls.

The strengths of the study includes the use of extensive exclusion criteria, the limited age range of the patients and the evaluation of the reviewed data by a specialist. The limitations of the study include small number of patients, single-center study design, and lack of data on sociocultural aspect of the study patients.

Organic factors must be evaluated in conjunction with environmental factors when evaluating fall risk in the elderly. There have been many studies investigating the concurrent use of multiple medications in patients with falls, and in the present study, patients were evaluated with tests that are used to evaluate predispositions to falls, and the findings may be relevant in efforts to come up with measures to decrease the risk of falls. The number of medications used by elderly patients may be reduced through lifestyle modifications, and the type and number of currently used medications must be taken into consideration when prescribing new medications. Monitoring vitamin D levels from early ages, and maintaining optimal levels may be important in maintaining a good neuromuscular structure in advanced ages. More comprehensive studies involving larger populations should be conducted.

Funding: There is no financial support and sponsorship

Conflict of Interest: The authors declare that they have no conflict of interest.

Ethical statement: Ethical approval was obtained from Clinical Trials Ethical Board at Bolu Abant Baysal University (Date and decision number: 2015/64).

Open Access Statement

This is an open access journal which means that all content is freely available without charge to the user or his/her institution under the terms of the Creative Commons Attribution NonCommercial License (http://creativecommons.org/licenses/bync/4.0). Users are allowed to read, download, copy, distribute, print, search, or link to the full 
texts of the articles, without asking prior permission from the publisher or the author.

\section{References}

[1]Wu TY, Chie WC, Yang RS, et al. Factors associated with falls among communitydwelling older people in Taiwan. Ann Acad Med Singap. 2013; 42(7):320-27.

[2]Moylan KC, Binder EF. Falls in older adults: risk assessment, management and prevention. Am J Med. 2007; 120(6):493.e16.

[3]Richardson K, Bennett K, Kenny RA. Polypharmacy including falls riskincreasing medications and subsequent falls in community-dwelling middle-aged and older adults. Age Ageing. 2015; 44(1):9096.

[4]Baranzini F, Diurni M, Ceccon F, et al. Fallrelated injuries in a nursing home setting: is polypharmacy a risk factor? BMC Health Serv Res. 2009; 9:228.

[5]Hammond T, Wilson A. Polypharmacy and falls in elderly: A literatüre review. Nurs Midwifery Stud. 2013; 2(2):171-75.

[6]Kalyani RR, Stein B, Vliyil R, et al. Vitamin $\mathrm{D}$ treatment for the prevention of falls in older adults: Systematic review and metaanalysis. J Am Geriatr Soc. 2010; 58(7):1299-310.

[7]Ziere G, Dieleman JP, Hofman A, et al. Polypharmacy and falls in the middle age and elderly population. B J Clin Pharmacol. 2005;61(2):218-23.

[8]Ambrose AF, Paul G, Hausdorff JM. Risk factors for falls among older adults: A review of the literatüre. Maturitas. 2013;75(1):51-61.

[9]Kojima T, Akishita M, Nakamura T, el al. Association of the polypharmacy with fall risk among geriatric outpatients. Geriatr Gerontol Int. 2011;11(4):438-44.
[10]Zia A, Kamaruzzzaman SB, Tan MP. The consumption of two or more fallriskincreasing drugs rather than polypharmacy is associated with falls. Geriatr Gerontol Int. 2017; 17(3):436-70.

[11]Skalska A, Gałaś A, Grodzicki T. 25hydroxyvitamin $\mathrm{D}$ and physical and cognitive performance in older people with chronic conditions. Pol Arch Med Wewn. 2012;122(4):162-69.

[12]Pan HH, Li CY, Chen TJ, et al. Association of polypharmacy with fall-related fractures in older Taiwanese people: age- and genderspecific analyses. BMJ Open. 2014;4(3):e004428.

[13]Halfon M, Phan O, Teta D. Vitamin D: a review on its effects on muscle strength, the risk of fall, and frailty. Biomed Res Int. 2015; 2015:953241.

[14]Romagnoli E, Pepe J, Piemonte S, et al. Management of endocrine disease: value and limitations of assessing vitamin D nutritional status and advised levels of vitamin D supplementation. Eur J Endocrinol. 2013; 169(4):59-69.

[15]Fried TR, O'leary J, Towle V, et al. Health outcomes associated with polypharmacy in Community Dwelling older adults: A systematic review J Am Geriatr Soc. 2014;62(12):2261-72.

[16]Balaban Ö, Nacir B, Erdem HR, et al. Evaluation of balance function. J PM\&R Science. 2009; 12:133-39.

[17] Vellas BJ, Wayne SJ, Romero L, et al. Oneleg balance is an important predictor of injurious falls in older persons. J Am Geriatr. Soc. 1997;45(6):735-38.

[18]Dhalwani NN, Fahami R, Sathanapally H, et al. Association between polypharmacy and falls in older adults: a longitudinal study from England. BMJ Open. 2017;7-e016358. 
[19]Laflamme L, Monárrez-Espino J, Johnell K, et al. Type, number or both? A populationbased matched case-control study on the risk of fall injuries among older people and number of medications beyond fall-inducing drugs. PloS One. 2015; 10(3):-e0123390.

[20]Ham AC, Swart KM, Enneman AWI, et al. Medication-related fall incidents in an older, ambulant population: the B-PROOF study. Drugs Aging. 2014; 31(12):917-27.

[21]Bischoff-Ferrari HA, Giovannucci E, Willett WC, et al. Estimation of optimal serum concentrations of 25-hydroxyvitamin D for multiple health outcomes. Am J Clin Nutr. 2006;84(1):18-28. 